\title{
Consumo calórico, estado nutricional materno, y retraso del crecimiento intrauterino
}

\author{
Energy intake, maternal nutritional status \\ and intrauterine growth retardation
}

Eliana Bender Martins 1

Rosa María Núñez Urquiza 2

\footnotetext{
1 Faculdade de Nutrição, Universidade Federal de Pelotas. C. P. 354 , Pelotas, RS 96010-900 ebender@ufpel.tche.br 2 Centro de Investigación en Sistemas de Salud, Instituto Nacional de Salud Pública. Av. Universidad 655, Cuernavaca, Morelos 62508, México. rmanunez@insp.mx
}

\begin{abstract}
To explore the association between low caloric intake during pregnancy and intrauterine growth retardation (IUGR), a case-control study with 264 cases and 892 controls was conducted in three maternity hospitals in Mexico City from January to August 1995. Nutritional information on pregnancy was recorded using a previously validated food frequency questionnaire. After adjusting for other known maternal risk factors in the non-conditional logistic regression analysis, for the total sample, caloric intake did not present a direct effect on IUGR (OR: 0.99; CI 95\%: 0.99-1.00). However the odds ratios were as follows for women: with low pre-gestational weight (OR: 2.31; CI 95\%: 1.59-3.36), in first gestation (OR: 1.72; CI 95\%: 1.18-2.51), with low birth weight infants (OR: 3.54; CI 95\%: 1.93-6.46), and presenting hypertension during pregnancy (OR: 1.61; CI 95\%: 1.00-2.59).
\end{abstract}

Key words Fetal Growth Retardation; Mother Nutrition; Energy Intake

Resumen Para conocer la asociación entre el consumo de energía en el embarazo y el retraso del crecimiento intrauterino (RCIU), se llevó a cabo un estudio de caso-cohorte en tres hospitales de maternidad de la ciudad de México, entre enero y agosto de 1995. De un total de 4.000 partos que ocurrieron en este período, nos referimos en este estudio a los resultados de un análisis de 264 casos de RCIU y 892 controles. Se procedió a un análisis de regresión logística no condicionada, en el que también se ajustaron los factores maternos potencialmente proclives a la confusión. Para el total de los casos no se observó un efecto directo del consumo de energía en el RCIU (RM: 0,99; IC 95\%: 0,99-1,00). Sin embargo, entre las mujeres que comenzaron el embarazo con un peso de 50 kilos o menos, el consumo de energía en relación a RCIU mostró una razón de momios de 2,31 (RM: 2,31; IC 95\%: 1,59-3,36); para las mujeres primigestas de 1,72 (RM: 1,72; IC 95\%: 1,18-2,51; en aquellas con antecedente de bajo peso al nacer de 3,54 (RM: 3,54; IC 95\%: 1,93-6,46) y para aquellas que presentaron tensión arterial alta en el embarazo de 1,61 (RM: 1,61; IC 95\%: 1,00-2,59).

Palabras clave Retardo del Crecimiento Fetal; Nutrición de la Madre; Ingestión de Energía 


\section{Introducción}

El retraso del crecimiento intrauterino (RCIU) constituye una importante causa del bajo peso al nacer en países en vías de desarrollo (De Onis et al., 1998; Soto et al, 1988). La trascendencia del RCIU se evidencia en su asociación con la morbi-mortalidad neonatal y en su influencia en el patrón de crecimiento postnatal de los niños (Villar et al., 1984). Estudios recientes sugieren que el RCIU es un factor asociado con las enfermedades cardiovasculares, la hipertensión y el diabetes tipo II del adulto (Godfrey \& Barker, 2000).

Mientras que en los países industrializados el factor más asociado con RCIU es el tabaquismo, en los países en vías de desarrollo, los factores nutricionales maternos son los más importantes en la etiología del RCIU (Kramer, 1987).

Según los informes de las estadísticas vitales para el año de 1992 en México, los trastornos relacionados con el bajo peso al nacer, el retraso del crecimiento intrauterino y pre-madurez ocuparon el segundo lugar dentro de las principales causas de defunción fetal, superados sólo por las anomalías congénitas (SSA, 1992).

En el presente artículo, se describen los resultados obtenidos en un estudio de tipo casocohorte, enfocándonos en el análisis de la asociación entre la ingestión calórica, el estado nutricional materno y su relación con el retraso del crecimiento intrauterino.

\section{Metodología}

El estudio original se diseñó con la finalidad de evaluar la exposición prenatal a plomo y su relación con el parto prematuro, bajo peso al nacer y retraso en el crecimiento intrauterino. De un total de 4.000 partos que ocurrieron en el período - de enero a agosto de 1995 - en tres hospitales de maternidad de la ciudad de México (Castelazo Ayala, Hospital Tlaltelolco, y Hospital de la Mujer), se identificaron 277 casos de retraso de crecimiento intrauterino y se efectuaron 1.113 controles. Sin embargo, la población del presente estudio quedó constituida sólo por aquellos casos y controles que contaron con información nutricional y antropométrica completa, por lo que la muestra final fue de 264 niños con retraso del crecimiento intrauterino y 892 controles.

Se consideraron como niños con RCIU todos aquellos con un peso al nacer por debajo del percentil 10 para su edad gestacional (Brenner et al., 1976). La edad gestacional se calculó con la fecha de la última menstruación que presentó la madre en el momento de la entrevista. Los controles, por otro lado, fueron realizados a niños con un peso al nacer acorde con su edad gestacional y con una edad gestacional mayor o igual a 37 semanas. Los criterios de exclusión: embarazos gemelares, residencia de la madre menor a un año en la Ciudad de México. Todos los participantes firmaron una carta de consentimiento informando de su disponibilidad para el estudio. Un pediatra perteneciente a la institución participante, debidamente capacitado y estandarizado según el método propuesto por Ballard (1991), fue responsable de la evaluación del recién nacido y la valoración de la edad gestacional. Un equipo del estudio previamente capacitado fue responsable de la selección de los casos, controles y la evaluación de la calidad de la información.

La información acerca de las características sociodemográficas, historia obstétrica, hábitos relacionados con el tabaquismo y alcohólicos, dieta, talla y peso en el preembarazo, se obtuvo a partir de un cuestionario estructurado, aplicado por entrevistadores debidamente capacitados, inmediatamente después de que las madres ingresaban en el hospital. La información del peso pregestacional y de la talla materna fue registrada según lo informado por la madre durante la entrevista.

El nivel socioeconómico fue construido de acuerdo al desarrollado por Bronfman et al. (1988); para clasificar el cuidado prenatal, se hizo operativo un Índice de Atención Prenatal, con base en el método desarrollado por Kessner et al. (1973). El estado nutricional materno fue evaluado mediante las siguientes variables: peso pregestacional, talla materna e índice de masa corporal (IMC). Se consideraron desnutridas, las mujeres que presentaban un peso pregestacional de $50 \mathrm{~kg}$ o menos; o una talla por debajo de $150 \mathrm{~cm}$ (Kramer, 1987), o tenían un índice de masa corporal menor que 19,8. El IMC fue categorizado según las recomendaciones del Subcommittee on Nutritional Status and Wheight During Pregnancy (1990), que clasifica el IMC como bajo $\left(<19,8 \mathrm{~kg} / \mathrm{m}^{2}\right)$, alto $\left(>26,0-29,0 \mathrm{~kg} / \mathrm{m}^{2}\right)$ y muy alto $\left(>29,0 \mathrm{~kg} / \mathrm{m}^{2}\right)$. Luego, se construyó una variable dicotómica, teniendo el IMC bajo como punto de corte para desnutrición.

Mediante la aplicación de un cuestionario semi-estructurado de frecuencia de consumo de alimentos previamente validado (Hernández et al., 1998) se obtuvo información de cada una de las mujeres acerca de la ingestión de 95 alimentos a lo largo de su embarazo. La frecuencia de consumo diario se hizo en base al tama- 
ño de una porción predeterminada y con 10 opciones de frecuencia que incluían desde "nunca" hasta "seis veces" al día. Asimismo, la ingestión calórica fue estimada mediante el uso del paquete computarizado Food Intake Analysis System (FIAS 3.0), que incluye información del contenido de nutrientes presentes en los alimentos registrados de manera similar a lo informado por otros (López-Carrillo et al., 1999). Para el análisis de los datos se utilizaron técnicas univariadas, pruebas simples de asociación y el modelo multivariado de regresión logística no condicional (Kleinbaum et al., 1988). Este último con la finalidad de obtener el mejor estimador del efecto de la ingestión calórica en la probabilidad de tener un neonato con retraso en el crecimiento intrauterino, controlado por las variables consideradas confusoras.

La variable dependiente, es decir el evento, fue el retraso del crecimiento intrauterino, considerado de manera dicotómica. La variable independiente de exposición fue el consumo habitual de energía durante el embarazo. Las variables independientes (confusores potenciales) del modelo saturado incluyeron el peso pregestacional, la talla materna, el estado civil, la edad, la escolaridad materna, la edad de la menarca, el nivel socioeconómico, la ocupación, el tabaquismo y el consumo de alcohol durante el embarazo, antecededentes de aborto y de bajo peso al nacer, el índice de atención prenatal, la primiparidad, la tensión arterial durante el embarazo. Posteriormente, y mediante la estadística de máxima verosimilitud, a través de la prueba del regresión logística, se fueron excluyendo las variables que no presentaron significación estadística en el modelo, hasta obtener un modelo con el menor número de variables. Para el análisis se utilizó el paquete estadístico STATA versión 4.0.

\section{Resultados}

La Figura 1, muestra la distribución del grupo de casos con RCIU con sus respectivas combinaciones; así tenemos que 102 casos (39\%) eran de bajo peso al nacer (BPN: $<2.500 \mathrm{~g})$, y solamente 17 casos (6\%), que además del bajo peso al nacer eran nacimientos pretérmino (PT: $<37$ semanas de embarazo).

Alrededor de la mitad de los casos de RCIU se caracterizó por no tener bajo peso al nacer ni ser partos pretérminos $(n=145)$.

En la Tabla 1 se presentan comparaciones de medias y de proporciones entre casos y controles de algunas variables socioeconómicas y de salud estudiadas en esta muestra.
Figura 1

Distribuición de casos de RCIU según peso al nacer y edad de gestación.

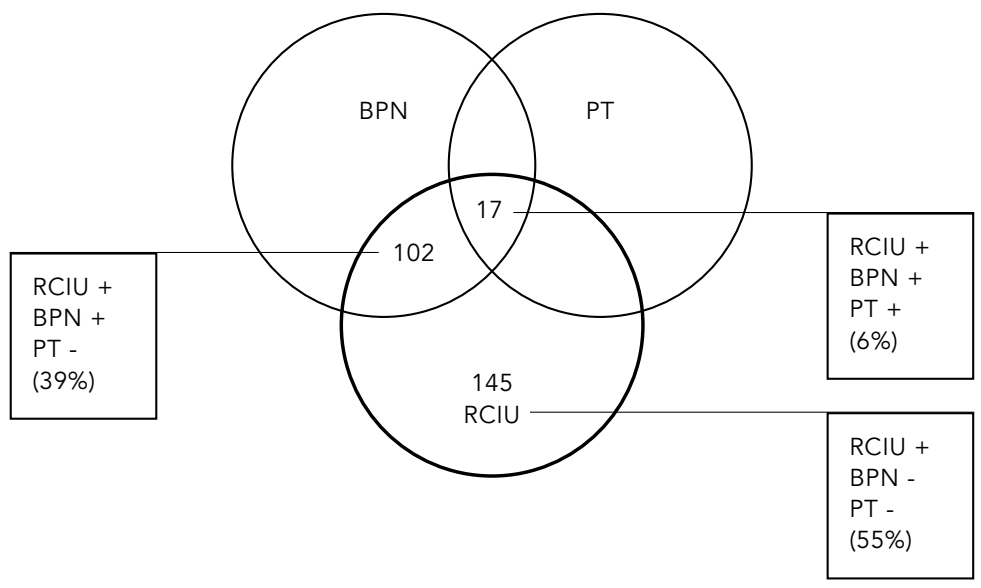

$\mathrm{RCIU}=$ retraso del crecimiento intrauterino; $\mathrm{BPN}=$ bajo peso al nacer; $\mathrm{PT}=$ pretérmino.

En relación a los indicadores nutricionales maternos, se encontró que el peso pregestacional de las maders de neonatos com RCIU $(53,37 \mathrm{~kg})$ y su IMC $(22,44)$, presentaron valores promedios significativamente más bajos, en comparación a los controles. Una proporción significativamente mayor de madres entre los casos (17\%), presentó episodio de hipertensión arterial durante el embarazo, así como del antecedente de hijos con bajo peso al nascer (16\%) en comparación a los controles $(12 \%$ y $6 \%$ respectivamente). En cuanto al número de hijos, había proporcionalmente más primigestas entre los casos (46\%), que entre los controles (40\%).

En relación a la escolaridad materna se observó que las madres tenían predominantemente de 7 a 12 años de escolaridad, aunque el grupo de las madres de casos era proporcionalmente mayor (73\%) comparado a los controles (66\%). En cuanto al nivel socioeconómico, no se observaron diferencias significativas entre casos y controles. En relación a la atención prenatal, se encontró en ambos grupos, una mayor proporción de madres que acudieron regularmente a los servicios de salud.

No se observaron diferencias significativas en las demás variables, tales como el hábito del tabaquismo durante el embarazo, el consumo de bebidas alcohólicas, la edad materna, la edad de la menarca, la ocupación materna (no se muestran los resultados).

Con respecto al consumo energético promedio durante el embarazo, no se encontraron 
Principales características entre casos de RCIU y controles. Ciudad de México, 1995.

\begin{tabular}{|c|c|c|c|c|c|}
\hline \multirow[t]{2}{*}{ Variables } & \multicolumn{2}{|c|}{ RCIU1 } & \multicolumn{2}{|c|}{ Controles } & \multirow[b]{2}{*}{$p^{2}$ ttest } \\
\hline & media & ds & media & ds & \\
\hline \multicolumn{6}{|l|}{ Antropométricas } \\
\hline Peso pregestacional2 & 53,37 & 9.89 & 56.27 & 9.14 & 0,000 \\
\hline IMC 3 pregestacional $\left(\mathrm{kg} / \mathrm{m}^{2}\right)$ & 22,44 & 3.9 & 23.41 & 3.6 & 0,002 \\
\hline Talla materna $4(\mathrm{~cm})$ & 154,61 & 6.6 & 155.82 & 6.66 & 0,018 \\
\hline Reproductivas y Socioeconómicas & $\mathbf{n}$ & $\%$ & $\mathbf{n}$ & $\%$ & $\mathbf{p}^{2} \mathrm{chi}^{2}$ \\
\hline \multicolumn{6}{|l|}{ Reproductivas } \\
\hline Hipertensión en el embarazo5 & 46 & 17 & 108 & 12 & 0,027 \\
\hline Antecedentes de BPN6 & 43 & 16 & 54 & 6 & 0,000 \\
\hline Primiparidad7 & 123 & 46 & 355 & 40 & 0,049 \\
\hline \multicolumn{6}{|l|}{ Socioeconómicas } \\
\hline \multicolumn{6}{|l|}{ Nivel socioeconómico8 } \\
\hline Bajo & 55 & 22 & 192 & 22 & 0,680 \\
\hline Medio & 146 & 58 & 475 & 55 & \\
\hline Alto & 52 & 20 & 198 & 23 & \\
\hline \multicolumn{6}{|l|}{ Escolaridad Materna9 } \\
\hline Hasta 6 años & 53 & 20 & 218 & 25 & 0,055 \\
\hline De 7 a 12 años & 192 & 73 & 580 & 66 & \\
\hline más de 12 años & 16 & 6 & 82 & 9 & \\
\hline \multicolumn{6}{|l|}{ Atención Prenatal10 } \\
\hline Adecuada & 63 & 25 & 205 & 24 & 0,410 \\
\hline Regular & 118 & 46 & 431 & 50 & \\
\hline Inadecuada & 75 & 29 & 219 & 26 & \\
\hline
\end{tabular}

$1 \mathrm{RCIU}=$ retraso del crecimiento intrautuerino (Brenner et al., 1976); 2 Peso recordatorio previo al embarazo o en el primer mes de gestación $(n=984) .3$ IMC = Índice de Masa Corporal $(n=807)$;

4 Talla reportada $(n=856) ; 5$ Hipertensión reportada por la madre según el diagnóstico médico $(n=1.153)$; $6 \mathrm{BPN}=$ bajo peso al nacer $(<2,500 \mathrm{~g} ; \mathrm{n}=1.156) ; 7$ Primiparidad comparada a la multiparidad $(\mathrm{n}=1.156)$;

8 Nivel socioeconómico según el Índice de Bronfman. 9 Escolaridad materna en años completos $(n=1.141)$

10 Índice de Atención Prenatal según los criterios de Kessner $(n=1.111)$.

diferencias significativas entre los casos y los controles (2.004 y 1.998kcal respectivamente); Entre las 984 mujeres de las que se contaba con información sobre el peso pregestacional, 316 (32\%) fueron clasificadas como desnutridas (peso $\leq 50 \mathrm{~kg}$ ); de estas 101 (10\%) tuvieron hijos con RCIU (no se presentan los datos).

Al caracterizar el estado nutricional según el Índice de Masa Corporal, una menor proporción de mujeres, (15\%) fue clasificada como desnutrida (IMC < 19,80); de estas $6 \%$ tuvieron hijos con RCIU;

En la Tabla 2 se describe el modelo final, por el cual se observa que no hubo efecto del consumo calórico en la incidencia del RCIU. Asimismo, se observa el efecto de las demás variables para la presencia del RCIU; como en las mujeres desnutridas (peso $\leq 50 \mathrm{~kg}$ ) que presen- taron 2,12 veces más riesgo para el RCIU, (RM 2,12; IC 95\%: 1,53-2,92). Las primigestas y las que tuvieron el antecedente de hijo con bajo peso al nacer presentaron un mayor riesgo de tener hijos con RCIU (RM: 1,76; IC 95\%: 1,262,48 ) y RM: 3,97; IC 95\%: 2,35-6,71). Las mujeres que manifestaron episodios de presión arterial elevada durante el embarazo, también presentaron un mayor riesgo de tener hijos con RCIU (RM: 1,54; IC 95\%: 1,00-2,36).

\section{Discusión}

Al igual que lo reportado por otros estudios (De Onis, 1998; Horta, 1997), alrededor de la mitad de los casos de RCIU no eran pretérmino ni de bajo peso al nacer. Este hallazgo sugiere que el 
Modelo Multivariado de regresión logística para el efecto del consumo de energía en el RCIU.

\begin{tabular}{lcc}
\hline Variable & Razón de momios & Intervalo de confianza al 95\% \\
\hline Consumo de energía1 & 1,00 & $0,99-1,00$ \\
$\begin{array}{l}\text { Desnutrición2 } \\
\text { Índice de Atención Prenatal3 }\end{array}$ & 2,12 & $1,53-2,92$ \\
$\quad$ Regular & 0,86 & $0,59-1,27$ \\
$\quad$ Inadecuado & 1,06 & $0,69-1,65$ \\
Presión elevada durante el embarazo4 & 1,54 & $1,00-2,36$ \\
Primiparidad & 1,76 & $1,26-2,48$ \\
Antecedente de hijo con BPN & 3,97 & $2,35-6,71$ \\
\hline
\end{tabular}

1 Energía: analizada como variable continua.

2 Desnutrición según la variable: peso materno $\leq 50 \mathrm{~kg}$

3 Índice de atención Prenatal: según el Índice de Kessner.

4 Presión arterial elevada: según el diagnóstico médico referido a las mujeres (sí o no).

$\mathrm{RCIU}=$ retraso del crecimiento intrauterino; $\mathrm{BPN}=$ bajo peso al nacer.

RCIU es un indicador insustituible para estimar la real magnitud del problema de desnutrición al nacer.

El consumo energético diario promedio encontrado $(2.000 \mathrm{kcal} / \mathrm{d})$ fue similar al presentado por otros estudios (Durnin, 1987; Rush et al., 1980). La Encuesta Nacional de Nutrición México (1988) mostró un promedio de consumo energético de $1.768 \mathrm{kcal} / \mathrm{d}$ entre las mujeres embarazadas (Flores et al., 1998). Asimismo, en otros estudios se manifiestan promedios más altos que el encontrado por nosotros, como, por ejemplo, el de Escocia $(2.120 \mathrm{kcal} / \mathrm{d})$ y el de la zona rural de México (2.356kcal/d); (Durnin, 1987; Martínez et al., 1994). En los estudios mencionados, se utilizó predominantemente el método recordatorio de 24 hs durante un día; mientras que, en este estudio, se utilizó el método de frecuencia de consumo diario.

Como una de las principales ventajas de este método se menciona la capacidad de captar la variabilidad diaria de la ingestión dietética en cada individuo. Y con eso, se espera haber reunido información más representativa del consumo de energía y demás nutrientes entre las embarazadas de esta muestra. También fue posible establecer comparaciones entre los grupos que más ingirieron calorías frente a los que menos lo hicieron. Además, este método posibilitó evaluar la asociación entre los grupos según la intensidad de consumo y el RCIU.

Cuando se estratificaron los grupos en cuartiles según la intensidad de consumo, se observó que los valores más bajos de consumo oscilaron de 720 hasta $1.562 \mathrm{kcal}$. Tomando en cuenta lo referente a las Recomendaciones del Subcomité de Ingestión Dietética y Suplemen- tación durante el Embarazo, el hecho de que un consumo promedio de nutrientes no alcance lo recomendable, no necesariamente implica una deficiencia en un determinado nutriente; por lo tanto, no se esperaría que todas las mujeres cuyo consumo energético fue bajo tuvieran hijos con RCIU; aun así, se esperaría una mayor incidencia de RCIU entre las 47 mujeres desnutridas que mostraron los valores más bajos de consumo energético. De éstas, apenas 10 (21\%) tuvieron hijos con RCIU.

Con respecto a los demás factores de riesgo encontrados en este estudio, conviene destacar que el antecedente de hijos con bajo peso al nacer además de ser un importante predictor del riesgo para el RCIU, refleja condiciones anteriores a este embarazo, que tuvieron como consecuencia el bajo peso de sus productos; por eso puede ser también considerado un marcador de riesgo. En cuanto a la paridad, los resultados encontrados en este estudio coinciden con los hallazgos de otros estudios donde también se encontró asociación entre primiparidad y mayor riesgo para el RCIU (Kramer, 1987). Aunque el papel de la paridad todavía no esté bien establecido debido a la influencia de la edad en la paridad, Shoham-Vardi et al. (1994), encontraron un efecto independiente de la primiparidad en el RCIU (RM:1,62, IC 95\%: 1,30-2,01).

La hipertensión arterial durante el embarazo, aunque aquí presentara un riesgo marginal, es un factor bien evidenciado en otros estudios, como de riesgo para el RCIU (Molina, 1998).

Con relación al Índice de Atención Prenatal, pese a que no fue posible hacer una discriminación adecuada por estratos, puesto que el grupo presentaba características homogéneas, 
los resultados sugieren la importancia de la atención al parto para el desarrollo adecuado del neonato.

En cuanto a la clasificación del estado nutricional de las mujeres, este se basó en las medidas antropométricas reportadas por las madres, teniendo en cuenta que son medidas generalmente aceptadas en otros estudios (Willet, 1990). En un estudio realizado en una muestra de funcionarios de un banco estatal en Brasil, Chór et al., (1999) encontraron un coeficiente de correlación intra clase de 0,98 para los valores de peso reportado comparado a la medición directa, y de 0,95 para el IMC, confirmando la alta correlación entre el peso reportado y la medida directa.

\section{Agradecimientos}

A la Dra. Tereza González-Cossío, por sus valiosas sugerencias en el campo de la Nutrición; a los investigadores Mario Flores Aldana y Víctor Tovar Guzmán, del Centro de Investigación en Salud Poblacional; al Dr. Malaquías López-Cervantes, del Centro de Investigación en Sistemas de Salud, Instituto Nacional de Salud Pública, México, quien concedió la base de los datos, posibilitando la elaboración de esta investigación y al Consejo Nacional de Ciencia y Tecnología, por el apoyo recibido durante el período de estudio.
En cuanto al Nivel Socieconómico, los grupos estaban similarmente distribuidos entre los estratos, aun así se observó una mayor proporción de madres en el nivel socioeconómico medio.

Finalmente y para concluir, este estudio posibilitó constatar que, entre las mujeres embarazadas que buscan los servicios de atención prenatal de la ciudad de México, la mujeres más expuestas a tener un hijo con RCIU son aquellas que además de presentar un bajo peso antes del embarazo, son primigestas, o tuvieron antecedente de hijo con retraso del crecimiento intrauterino, o sufren elevación de la tensión arterial durante el embarazo, y constituyen uno de los grupos de mayor potencial de beneficio en las acciones de promoción y protección a la salud.

\section{Referencias}

BALLARD, J. L.; KHOURY, J. C.; WEDIG, K.; WANG, L.; EILERS-WALSMAN, B. L. \& LIPP, R., 1991. New Ballard Score expanded to include extremely premature infants. Journal of Pediatrics, 119:417-423.

BRENNER, W. E.; EDELMAN, D. A. \& HENDRICKS, C. H., 1976. A standard of fetal growth for the United States of America. American Journal of Obstetrics and Gynecology, 126:555-564.

BRONFMAN, M.; GUISCAFRÉ, H.; CASTRO, V. \& GUTIÉRREZ, G., 1988. La medición de la desigualdad: Una estrategia metodológica; Análisis de las características socioeconómicas de la muestra. México. Archivos de Investigación Médica, 19: 351-360.

CHÓR, D.; COUTINHO, E. S. F. \& LAURENTI, R., 1999. Reliability of self-reported weight and height among State bank employees. Revista de Saúde Pública, 33:16-23.

COMMITTEE ON NUTRITIONAL STATUS DURING PREGNANCY AND LACTATION, INSTITUTE OF MEDICINE, 1990. Nutrition During Pregnancy. Washington, DC: National Academy Press.

DURNIN, J. V., 1987. Energy requirements of pregnancy: An integration of the longitudinal data from the five-country study. Lancet, 14:1131-1133. 
DE ONIS, M.; BLOSSNER, M. \& VILLAR, J., 1998. Levels and patterns of intrauterine growth retardation in developing countries. European Journal of Clinical Nutrition, 52:5-15.

FLORES, M.; MELGAR, H.; CORTES, C.; RIVERA, M.; RIVERA, J. \& SEPULVEDA, J., 1998. Consumo de energía y nutrimentos en mujeres mexicanas en edad reproductiva. Salud Pública de México, 40: 161-171.

GODFREY, K. M. \& BARKER, D. J., 2000. Fetal nutrition and adult disease. American Journal of Clinical Nutrition 71:1344-1352.

HERNANDEZ-AVILA, M.; ROMIEU, I.; PARRA, S.; HERNANDEZ-AVILA, J.; MADRIGAL, H. \& WILLET, W., 1998. Validity and reproducibility of a food frequency questionnaire to assess dietary intake in women living in Mexico City. Salud Pública de México, 40:133-140.

HORTA, B. L.; VICTORA, C. G.; MENEZES, A. M.; HALPERN, R. \& BARROS, F. C., 1997. Low birthweight, preterm births and intrauterine growth retardation in relation to maternal smoking. Paediatric and Perinatal Epidemiology, 11:140-151.

KESSNER, D. M.; SINGER, J.; KALK, C. E. \& SCHLESINGER, E. R., 1973. Infant Death: An Analysis by Maternal Risk and Health Care. Washington, DC: Institute of Medicine, National Academy of Sciences.

KLEINBAUM, D. G.; KUPPER, L. L. \& MULLER, K. E., 1988. Applied Regression Analysis and Other Multivariable Methods. Boston: PWS-Kent Publishing Co.

KRAMER, M. S., 1987. Determinant of low birth weight: methodological assessment and meta-analysis. Bulletin of World Health Organization, 65:663737.

LOPEZ-CARRILLO, L.; LOPEZ-CERVANTES, M.; WARDMARY, H.; BRAVO-ALVARADO, J. \& RAMIREZ-ESPITIA, A., 1999. Nutrient intake and gastric cancer in Mexico. International Journal of Cancer, 83:601-605.
MARTINEZ, H.; ALLEN, L.; LUNG, M.; CHAVEZ, A. \& PELTO, G., 1994. Maternal fatness in mexican women predicts body composition changes in pregnancy and lactation. In: Nutrient Regulation During Pregnancy, Lactation, and Infant Growth: Advances in Experimental Medicine and Biology, (L. Allen, J. King \& B. Lonnerdal, ed.), pp. 99-107, New York: Plenum Press.

MOLINA, M.; CASANUEVA, V.; PEREZ, R.; FERRADA, C.; CISTERNAS, J.; CID, L. \& SAEZ, K., 1998. Impact of hypertensive disease of pregnancy on intrauterine growth retardation. Revista Médica Chilena, 126:375-382.

RUSH, D.; STEIN, Z. \& SUSSER, M., 1980. Diet in Pregnancy: A Randomized Controlled Trial of Prenatal Nutritional Supplementation. New York: Alan Liss.

SHOHAM-VARDI, I.; LEIBERMAN, J. R. \& KOPERNIK, G., 1994. The association of primiparity with intrauterine growth retardation. European Journal of Obstetric \& Gynecology Reproductive Biology, 53:95-101.

SOTO, C. I.; BOBADILLA, J. L. \& MIRELES, P. C., 1988. Valores de referencia para evaluar el crecimiento intrauterino en nacimientos ocurridos en la ciudad de México. Salud Pública de México,1:68-80.

SSA (Secretaría de Salud), 1992. Estadísticas de Mortalidad. México, DF: SSA.

VILLAR, J.; SMERIGLIO, V.; MARTORELL, R.; BROWN, C. H. \& KLEIN, R. E., 1984. Heterogeneous growth and mental development of intrauterine growthretarded infants during the first 3 years of life. $P e$ diatrics, 74:783-791.

WILLETT, W., 1990. Anthropometric measure and body composition. In: Nutritional Epidemiology. (W. Willett, ed.), pp. 217-220, New York/Oxford: Oxford University Press.

Recibido el 28 de septiembre de 2001

Versión final presentada el 12 de julio de 2002

Aprobado el 9 de septiembre de 2002 